\title{
Prasugrel oder Clopidogrel nach STEMI?
}

\section{Hintergrund}

Goldstandard bei der Therapie eines STHebungsinfarktes (STEMI) ist neben der mechanischen Reperfusion die duale Plättchenhemmung mit Acetylsalicylsäure (ASS) und Clopidogrel. In einer aktuellen Studie wurden Wirksamkeit und Sicherheit von Prasugrel, einem neuen Thienopyridin, und Clopidogrel miteinander verglichen.

\section{Methoden}

In der TRITON-TIMI 38 (Trial to Assess Improvement in Therapeutic Outcomes by Optimizing Platelet Inhibition with Prasugrel-Thrombolysis in Myocardial Infarction) genannten Multicenter-Studie erhielten 3534 Patienten nach STEMI und perkutaner Koronarintervention zusätzlich zu ASS doppelblind und randomisiert entweder Prasugrel $(60 \mathrm{mg}$ Loading-Dosis und $10 \mathrm{mg}$ tägliche $\mathrm{Er}$ haltungsdosis; $\mathrm{n}=1769$ ) oder Clopidogrel $(300 \mathrm{mg}$ Loading-Dosis und $75 \mathrm{mg}$ Erhaltungsdosis; $n=1765)$. Primärer
Endpunkt war die Kombination aus Tod infolge kardiovaskulärer Ursache sowie nichttödlichem Myokardinfarkt oder Apoplex. Die Auswertung erfolgte auf Intention-to-treat-Basis. Das Follow-up betrug insgesamt 15 Monate mit einer Zwischenauswertung nach 30 Tagen.

\section{Ergebnisse}

Im Vergleich zu Clopidogrel verringerte Prasugrel nach STEMI und perkutaner Koronarintervention das Risiko ischämischer Ereignisse, ohne das Blutungsrisiko entscheidend zu erhöhen. Nach 30 Tagen war bei 115 Personen (6,5\%) mit Prasugrel und bei 166 Personen (9,5\%) mit Clopidogrel der primäre Endpunkt eingetreten (Hazard ratio 0,68; 95\%-Konfidenzintervall [KI] 0,54-0,87; $\mathrm{p}=0,0017)$. Dieser Effekt hielt auch nach 15 Monaten an (174 [10,0\%] versus 216 [12,4\%]; Hazard ratio 0,79; 95\%-KI 0,650,$97 ; p=0,02$ ). Die Number-needed-totreat lag bei 35 für den Zeitraum 30 Tage und 41 für die gesamte Studiendauer.
Die Anzahl der unabhängig von koronaren Bypassoperationen auftretenden TIMI-Major-Blutungen unterschied sich nicht zwischen den beiden Gruppen nach 30 Tagen $(p=0,36)$ und nach 15 Monaten $(p=0,65)$. Auch die Zahl lebensbedrohlicher TIMI-Blutungen sowie von TIMI-Minor-Blutungen war vergleichbar. Lediglich die Rate von TIMI-Major-Blutungen nach koronarer Bypassoperation war in der PrasugrelGruppe signifikant erhöht $(\mathrm{p}=0,003)$.

\section{Fazit}

In den ersten 15 Monaten nach STEMI und perkutaner Koronarintervention sei die Kombination von ASS plus Prasugrel der Kombination ASS plus Clopidogrel in bezug auf die Reduktion kardiovaskulärer Ereignisse überlegen, ohne dabei das Blutungsrisiko entscheidend zu erhöhen, so die Autoren.

Dr. med. Winfried Keuthage, Münster

Quelle: Montalescot G, Wiviott SD, Braunwald E et al. Prasugrel compared with clopidogrel in patients undergoing percutaneous coronary intervention for ST-elevation myocardial infarction (TRITON-TIMI 38): double-blind, randomised controlled trial. N Engl ] Med 2009; 373: 723-731 